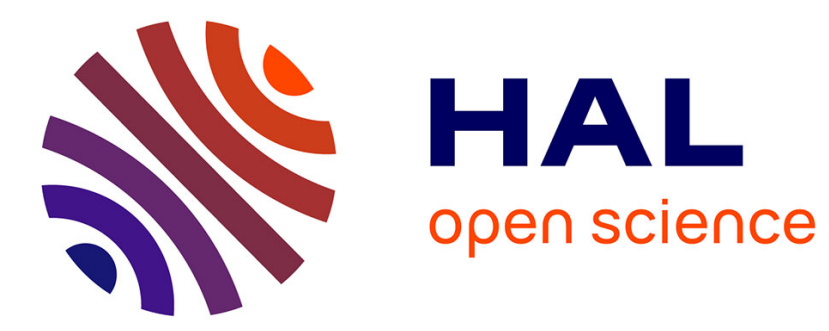

\title{
Rythm'blues: Accords et désaccords entre un bébé et sa mère déprimée.
}

Ouriel Rosenblum

\section{To cite this version:}

Ouriel Rosenblum. Rythm'blues: Accords et désaccords entre un bébé et sa mère déprimée.. Champ Psychosomatique, 2009, "Au commencement était le rythme.. ", 2 (54), pp.81 - 90. 10.3917/cpsy.054.0081 . hal-01516998

\section{HAL Id: hal-01516998 \\ https://hal.science/hal-01516998}

Submitted on 24 Jun 2017

HAL is a multi-disciplinary open access archive for the deposit and dissemination of scientific research documents, whether they are published or not. The documents may come from teaching and research institutions in France or abroad, or from public or private research centers.
L'archive ouverte pluridisciplinaire HAL, est destinée au dépôt et à la diffusion de documents scientifiques de niveau recherche, publiés ou non, émanant des établissements d'enseignement et de recherche français ou étrangers, des laboratoires publics ou privés. 


\title{
Rythm'blues : \\ Accords et désaccords entre un bébé et sa mère déprimée.
}

\author{
Ouriel Rosenblum
}

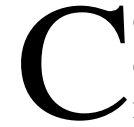

onscients des risques auxquels sont exposés les bébés dont la mère est déprimée au moment de leur naissance, et désireux de repérer précocement les dysharmonies précoces, nous avons entrepris une recherche longitudinale en étudiant les effets de cette dépression précoce (O. Rosenblum \&al.)

L'une des originalités fondamentales de la psychopathologie du nourrisson est son interdépendance du fonctionnement psychique de la mère. L'approche de l'enfant devant passer par sa relation à la mère, l'analyse de l'interaction devient un objet central d'étude, d'où la nécessité d'une vision bi-focale continue. Le défi de la psychopathologie du premier âge tient à notre capacité d'assumer cette dialectique méthodologique fondamentale respectant simultanément les caractéristiques individuelles du bébé et celles de la relation. En prenant comme fil rouge le rythme et ses variations entre les deux partenaires, nous allons tenter de décrire deux types interactifs retrouvés en fonction des caractéristiques de la dépression maternelle, en ne préjugeant pas sur l'aspect de la signification de cette dépression. Ainsi nous présenterons deux situations lorsque les bébés ont 3 mois en insistant sur la difficulté pour les partenaires de l'interaction de s'accorder en rythme. 
MARTHE 3 MOIS ET SA MAMAN DÉPRIMÉE ANXIEUSE

La mère est mal à l'aise lorsqu'elle porte son bébé, elle change constamment de position sans parvenir à un état confortable et stable. Sa fille s'agite et pleure fréquemment ; la mère ne parvient pas à la calmer. Ainsi, elle éprouve des difficultés à percevoir et à interpréter de manière adéquate les signaux émis par le bébé ; en effet, elle ne les anticipe pas, en revanche, elle tente de mettre en place un comportement d'essais successifs et d'erreurs répétées. Par ailleurs, elle utilise fréquemment, et ce, à contre temps, des objets tels des jouets ou des peluches pour tenter de médiatiser, de manière intrusive, des sollicitations inappropriée aux capacités du bébé d'y répondre. Ainsi, les réactions maternelles, la discontinuité des interactions entraînent souvent une protestation active de la part de Marthe avec détournement du regard, raidissement de la posture avec mise en hypertonie du corps, mouvements actifs des mains, des jambes, repoussant les tentatives anxieuses de la mère. Celle-ci intervient avec une précipitation inquiète vis-à-vis de l'attitude du bébé à son égard, faute d'être en mesure de l'anticiper. Ainsi, elle nous donne à voir son désarroi, renforçant son mouvement dépressif, de ne pouvoir entrer en empathie avec Marthe. Ici, on peut percevoir le débordement émotionnel de cette mère : elle stimule son bébé à l'excès, manifestant des signes de désorganisation qui nous laissent penser que les partenaires sont désaccordés. Cependant, Marthe peut néanmoins protester, laissant entrevoir une part active de sa part.

\section{PIERRE 3 MOIS ET SA MAMAN DÉPRIMÉE}

\section{RALENTIE}

D'emblée, on est frappé par la monotonie et la pauvreté affective de l'échange au cours duquel la mère n'affiche aucun signe de plaisir dans la rencontre avec son enfant avec lequel engage des tentatives de contacts corporels assez maladroites. L'ensemble de la séquence interactive dégage un sentiment de malaise se communiquant à l'observateur. La mère éprouve, en outre, des difficultés à percevoir les états d'éveil du bébé, ses capacités toniques, sa disponibilité à 


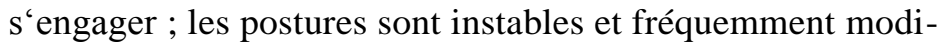
fiées par la mère, sa voix est très peu audible, la prosodie du discours maternel adressé à l'enfant est lente bien que modulée. Les séquences interactives indiquent qu'il existe destentatives maternelles pour entrer en interaction avec le bébé, mais qu'elles ne sont pas adaptées à ses capacités et s'accompagne d'une excitation visible de Pierre. La mère et son bébé, bien que présents, ne semblent pas communiquer en harmonie un contre temps s'est installé entre les deux partenaires, le tempo du rythme maternel étant davantage en lien avec le ralentissement psychomoteur imprimé par son vécu dépressif qu'accordé à la capacité d'entrer en relation de son bébé.

\section{DU BÉBÉ À L'EMPATHIE : UNE APPROCHE DES INTERACTIONS AFFECTIVES}

Pour forger nos propres outils, nous nous sommes plus spécifiquement penchés sur l'approche des interactions affectives, pont entre les représentations et les affects. Cette approche nous permettait de quitter le domaine strict des interactions limitées à l'étude des comportements, pour aborder le champ de la relation dans une perspective de l'écoute des processus psychiques précoces, prélude à l'approche psychothérapeutique.

Pourquoi choisir à nouveau le paradigme du bébé pour aborder le monde des émotions et par delà, la relation ?

A. Green nous a mis en garde contre le danger de choisir le paradigme de l'enfant qui nous ferait faire l'économie de l'opacité de la vie psychique, tout aveuglés par la perception de l'objet externe qu'est la mère, confondant l'infantile avec l'enfant La remontée aux origines, comme démarche explicative n'étant qu'une théorie sexuelle supplémentaire. Pourtant, selon nous, le bébé confronté à l'incapacité néonatale à agir, a besoin d'autrui, développe des liens qui s'étayent sur les besoins libidinaux, sexualisant la relation. Sa sensorialité appuyée par une capacité précoce d'expression deviendra le canal de l'action qui fournira alors la matière de l'interaction. 


\section{LA DÉPRESSION MATERNELLE COMME ILLUSTRATION D'UN DYSFONCTIONNEMENT INTERACTIF}

Notre étude a porté sur le suivi de dyades composées de mères déprimées et mères témoins pendant 2 ans. Les résultats nous indiquent, par la mesure de l'état d'engagement affectif (J.F. Cohn \& S.Campbell), que l'engagement affectif des mères déprimées est plus restreint que celui des mères témoins ainsi que celui des bébés des mères déprimées par rapport aux bébés de mères témoins. Les expressions faciales, vocales, les positions, les mouvements corporels et la direction du regard sont plus pauvres, exprimant un engagement affectif diminué de la part de deux partenaires de la dyade. Par la suite, nous avons affiné le type d'attachement « insecure » (M.D.S. Ainsworth et al.) engagés par les enfants à 1 an en fonction de l'aspect dimensionnel de la dépression maternelle (R. Jouvent et al.). Lorsque l'on compare le type d'attachement des enfants de mères déprimées, irritées, anxieuses avec celui des enfants de mères déprimées ralenties et émoussées, on constate que la proportion d'enfants « insécure-ambivalent » est prépondérante dans le premier groupe alors que celle des enfants « insécure-évitant» est dominante dans le deuxième groupe.

On peut faire l'hypothèse que les enfants qui ont engagé un «style » interactif avec une mère émoussée, plus indisponible affectivement, se sont accoutumés à une mère psychiquement absente au cours des interactions et ainsi ils se sont auto-organisés dans la relation sans l'appui d'une représentation maternelle étayante dans l'échange. Alors que les enfants qui ont engagé un «style » interactif avec une mère déprimée, anxieuse et irritée, souvent intrusive, peuvent mettre en place précocement un système défensif relativement efficace et souple, ce qui permet à l'enfant de se dégager d'une relation trop excitante et de pouvoir se réengager par la suite. L'anxiété maternelle témoignerait davantage d'un débordement affectif que d'un retrait et pourrait maintenir une relation où se mêle à la fois l'hyperstimulation maternelle, le retrait de l'enfant et le réajustement des deux partenaires. Aussi ces liens d'attachement seraient-ils plus une source d'ambivalence pour ces enfants de mères déprimées anxieuses que d'évitement. 
L'évaluation des expressions émotionnelles du bébé ainsi que l'état d'engagement affectif du partenaire au cours de l'interaction vient confirmer que l'interaction affective peut être un indicateur du développement de l'enfant aussi bien pour évaluer l'interaction que pour apprécier ultérieurement le type d'attachement que met en place l'enfant indiquant une intériorisation des patterns interactifs antérieurs.

L. Murray nous a montré que la qualité de l'engagement maternel avant dix-huit mois influence le fonctionnement cognitif de l'enfant à 5 ans. Ainsi les accordages avec les expressions affectives du bébé et leur élaboration répétitive pluri-modale servent à soutenir l'attention et l'engagement positif du bébé entraînant la construction de la cohérence de soi.

Le défi lancé lorsque l'on est ensemble, c'est la possibilité de changer sans mettre en péril la construction précédente. L'étude des interactions «dépressives» nous montre à l'inverse l'impossibilité pour la mère d'envisager toute modification, rigidifiant et appauvrissant l'interaction .

E. Fivaz propose, à partir des transitions observées au cours des interactions, de définir les bases d'un travail de déconstruction initiale virtuelle engagé par les partenaires afin d'anticiper les transformations imposées par les modifications interactives pour reconstruire une nouvelle figure qui contextualise déjà la suite.

Ainsi ces transformations successives qui se constituent au fur et à mesure font-elles naître une propriété émergente des invariants constitutifs des patterns interactifs.

\section{CONTRE-TRANSFERT ET «ÉNACTION»}

F. Varela, dans une perspective neurobiologique, va tenter définir la notion d'énaction. À partir de la notion d'action guidée par la perception et de la structure sensori-motrice du sujet, il nous présente la façon dont le sujet percevant est inscrit dans un corps et comment l'action peut être perceptivement guidée dans un monde qui dépend du sujet de la perception. Dans cette perspective, la perception aussi à l'énaction de ce monde environnant. Ainsi, comme le note M. Merleau-Ponty, l'organisme donne forme à son environnement en même temps qu'il est façonné par lui. L'idée d'énac- 
tion est celle selon laquelle voir des objets ne consiste pas à en extraire des traits visuels, mais à guider visuellement l'action dirigée vers eux.

Par ailleurs, dans une perspective psychanalytique, selon J. Sanville, à partir de nos contre-attitudes, 1 « enactment » semble pouvoir être traduit par «mise en jeu ».

À partir des théories de «l'énaction » développée par F. Varela, où la virtualité des schèmes d'action peut être une des voies des mouvements d'empathie, nous pourrions proposer une lecture des mouvements dépressifs maternels dans leur relation à l'enfant.

La mère déprimée se rend indisponible lors de l'engagement affectif dans la mesure où elle est hyperinvestie par l'affect dépressif qui envahit son champ relationnel. Ainsi elle maintient le cadre rigide interactif, afin d'utiliser le pôle fonctionnel des interactions comme guide de ses actes à l'égard des soins apportés au bébé. L'interaction ne peut alors subir des transformations qui désorganiseraient soit le pôle ralenti, soit le pôle anxieux de la dépression maternelle. À la rigidité du cadre interactif imposé par la mère, au cours du dialogue tonique, la contrepartie motrice et hypertonique de l'enfant apporte sa réponse interactive. Ici, l'irruption de l'affect dépressif maternel peut faire obstacle à une modulation de l'expression émotionnelle et contraint l'enfant à réguler ses états de tension interne par l'entremise de son système musculaire locomoteur proprioceptif, encore peu développé au début de la vie.

Ainsi les compétences précoces du bébé à lire les émotions du partenaire dans un bain d'affects sont utilisées plutôt à maintenir une régulation plus approximative de son système de pare-excitation à partir de sa tonicité.

On voit là que l'interruption des processus d' " énaction » maternelle à partir de l'envahissement affectif dépressif empêche paradoxalement la mise en place d'une empathie maternelle, gage de la virtualité d'un engagement autorisant des schémas anticipatifs de l'enchaînement interactif.

Dans ces premiers temps qui suivent la naissance et où elle se sent trop fragile, la mère n'a de cesse que de réduire la part d'incertitude dans ses relations avec son bébé. Les partenaires de la dyade mère déprimée-bébé sont-ils condamnés au visible de l'interaction qui, pour se maintenir, fait appel au présent concret qui peut se déployer lors des 
interactions précoces où la mère répète les mêmes actes au cours des soins quotidiens apportés au bébé.

Pour la mère, cette « concrétude » s'apparente à la fonctionnalité des soins apportés au bébé. Pour celui-ci, la rencontre interactive avec sa mère déprimée s'effectue à partir de la sensation tangible et palpable de la décharge tensionnelle motrice, figure pictographique de l'éprouvé interactif.

L'étude de la spécificité de l'interaction dépressive peut donc nous aider à comprendre que l'interaction mère-bébé n'a pas comme objectif ultime l'efficacité des comportements réciproques. Mais qu'à partir de l'accordage affectif éprouvé entre les partenaires, le défi interactif tenterait de se déployer, telle une portée musicale, à partir d'une narration partageable entre les parents et leur enfant qui serait issue de leur propre transmission intergénérationnelle. Ainsi la partition musicale jouée au cours de l'interaction s'emparerait également du livret écrit par la névrose infantile des parents, instants propices à l'interprétation de scénarii partagés, rejoués sur tous les modes, jusqu'à aboutir à une représentation fiable chez l'enfant de " l'être avec» ses partenaires.

Par ailleurs, l'un des intérêts de se pencher sur la vie émotionnelle et de ses avatars par l'étude des interactions affectives est de pouvoir repérer et de décrire les troubles affectifs chez le jeune enfant. Ainsi, la classification diagnostique concernant les troubles du très jeune enfant de 0 à 3 ans (S.I. Greenspan et al.) place au premier plan les troubles émotionnels dans la constitution du premier axe du diagnostic, ceci comportant aussi bien les troubles de l'anxiété, de l'humeur que ceux de l'expression affective ou bien de l'attachement. Les auteurs de cette nomenclature associent souvent ces perturbations à des dysharmonies interactionnelles entre le bébé et ses partenaires. Ainsi, actuellement, les classifications diagnostiques concernant les troubles constitués au cours de la première enfance privilégient l'axe affectif et interactionnel comme étant l'un des facteurs prépondérants pour la compréhension de l'apparition de ces troubles précoces. 


\section{OBSERVATION DES INTERACTIONS AFFECTIVES ET POUVOIR MÉTAPHORISANT DE L'EMPATHIE}

Pour un observateur, il est difficile d'observer, c'est-àdire recueillir des faits, sans les interpréter ; observer et penser sont donc pratiquement inséparables. Selon A. Nakov l'observateur a une fonction contenante de holding du «bébé à l'intérieur de la mère » et le bébé va souvent utiliser la présence de l'observateur avec le besoin d'être contenu et d'utiliser l'activité de pensée qu'un adulte met ainsi à sa disposition. Ainsi nous nous situons résolument dans une perspective où l'observation est très active et sert de mode de communication dans une démarche thérapeutique suivant l'approche clinique proposée par D. Winnicott. Lorsque nous sommes en présence des protagonistes de la dyade, les observateurs que nous sommes, semblent éprouver la nécessité de regarder comme si nous recherchions une confirmation de la réalité des interactions, comme si nous cherchions à revivre par procuration ce qui est, en fait, perdu pour nous. Par bonheur, cette réalité se dérobe sous nos yeux et dépend tout autant de l'organisation psychique de l'éprouvé que de la visée interprétative de l'observateur.

L'abord des interactions affectives nous permet peut être de nous dégager du regard porté et de nous laisser guider par notre capacité d'énaction c'est à dire la façon dont nous, sujets percevant, nous sommes inscrits dans un corps. Lorsque le bébé interagit avec ses partenaires nous pouvons nous laisser entraîner, notre corps réagit, créant un éprouvé ; mais l'action, qui possède des propriétés émergentes, n'est pas extériorisée. Notre action est perceptivement guidée dans un monde qui dépend du sujet de notre perception. Les affects, perceptions d'actions motrices (S. Freud), sont induits par les bébés affamés d'investissement où toutes leurs activités perceptives se situent dans un engagement global.

Notre action pourrait s'établir sur deux niveaux selon S. Lebovici :

- Le premier serait, par notre participation et notre regard, un surgissement d'une mise en scène corporelle de la dyade où l'on percevrait l'effet produit au sein de notre éprouvé corporel engagé. 
- Le deuxième permettrait, par notre contre-transfert et notre capacité de rêverie, véritable pouvoir de métaphorisation, la mise en récit de la rêverie maternelle qui elle, est destinée à un tiers. Ainsi, la traduction des affects en métaphores qui transmettent un investissement libidinal de la pensée, représente un acte créatif de nouveauté. L'énaction, mise en jeu, incarnerait alors le processus qui permettrait la création, la découverte de la métaphore chargée de sens pour tous les partenaires impliqués.

Notre compréhension affective émergente, proche de ce que Freud appelle les identifications primaires, mise en jeu à la fois par les affects de vitalité et l'autoérotisme du bébé est soutenue par l'attention conjointe des partenaires en présence. Selon S. Lebovici, ce moment émergeant constituerait notre empathie, processus intégratif du passage de l'Einsicht (voir en dedans) à celui d'Einfühlung (sentir au dedans).

Ainsi, la circulation entre le subjectif et ce qui est objectivement perçu, réalise une aire intermédiaire qui permet la constitution d'un espace potentiel où peut se jouer la relation. P. Fedida a décrit « cette capacité à laisser corporellement jouer en soi ce qui est vu et ce qui est entendu » chez l'analyste.

L'empathie, authentique démarche active, nous permet davantage de comprendre comment un bébé « se trouve avec » plutôt que de tenter d'objectiver et réifier le bébé. Audelà de cette accessibilité, l'empathie métaphorisante offre la possibilité de construire des objets porteurs de significations relationnelles qui libèrent de l'assujettissement aux choses non signifiantes, non transformables (H. Searles).

Ainsi les « signifiants énigmatiques » (J. Laplanche) suscitent-ils toujours nos interrogations potentielles quant au bébé que nous avons été. La rencontre du bébé avec ses parents nous confronte à l'originaire, lieu du refoulement. L'opacité de l'observation, en tant qu'objet d'étude, nous permet de percevoir le script joué par les protagonistes dans la relation. Les affects qui s'y déploient, suscitent notre empathie métaphorisante qui engendre une trame narrative.

Nous quitterions alors le champ de l'observable pour entrer dans celui de l'histoire. 


\section{BIBLIOGRAPHIE}

SAINSWORTH M.D.S., BLEHAR M.C., WATERS E., WALL S. (1978), Patterns of attachment : A psychological study of the Strange Situation, Hillsdale, NJ, Erlbaum.

COHN J.F., CAMPBELL S., REINALDO M., HOPKINS J. (1990), « Face to face interactions of post partum depressed and non depressed mother-infant pair at two months », Developmental Psychology, 26, 1, pp 15-24.

FÉDIDA P. (1978), «l'objeu » in L'absence, Paris, Gallimard-NRF, coll. «Connaissance de l'inconscient ».

FIVAZ-DEPEURSINGE E. (1995), La situation triadique, Intervention au Réseau INSERM, Bobigny, 15 sept. 1995.

FREUD S. (1921), «Psychologie collective et analyse du moi » In Essai de psychanalyse, Paris, Payot, 1968, pp 64-83.

GREEN A. (1979), «L'enfant modèle », Nouvelle Revue de Psychanalyse, 19, pp 27-48.

GREENSPAN S.I., WIEDER S. (1994), Diagnostic Classification of Mental Health and Developmental Disorders of Infancy and Early Childhood, Arlington, Zero to Three. National Center for Clinical Infant Programs Eds.

JOUVENT R., VINDREAU C., MONTREUIL M., BUNGENER C., WIDLÖCHER D. (1988), " La clinique pluridimensionnelle de l'humeur dépressive. Nouvelle version de l'échelle EHD»Psychiatrie Psychobiologique, 3, pp 245-253.

LAPLANCHE J. (1994), Nouveaux fondements pour la psychanalyse, Paris, Quadrige/ PUF.

LEBOVICI S. (1994), «Empathie et « enactment » dans le travail de contre-transfert » in Travail de contre-transfert et fonction contenante, Revue Française de Psychanalyse, pp 1551-1561.

MURRAY L. (1996), «Influence de la communication mère-bébé sur le développement psychologique de l'enfant »In Troubles relationnels père-mère-bébé : quels soins ?, ARIP-ER7S, pp 39-56.

NAKOV A. (1992), «La fonction observante de l'observateur», Psychanalyse de l'enfant, 14, pp 343-357.

ROSENBLUM O., CONQUY L., LATOCH J., HEMEURY-CUCKIER F., MAZET Ph. (2004), « La dépression maternelle du post-partum, figure paradigmatique d'un dysfonctionnement interactif affectif ", Perspectives Psychiatriques, 43, 3, pp 204-209.

SEARLES H. (1982), «Différenciation entre pensée concrète et pensée métaphorique », Nouvelle Revue de Psychanalyse, 25, pp 331-352.

VARELA F., THOMSON E., ROSCH E. (1993), L'inscription corporelle de l'esprit, sciences cognitives et expérience humaine, Paris, Seuil. 